\title{
Renal:Systemic Renin Indices and Renal Vein Renin Ratios as Prognostic Indicators in Remedial Renovascular Hypertension ${ }^{1}$
}

\author{
JAMES C. STANLEY, M.D., ${ }^{2}$ BRUCE L. GEWERTZ, M.D., AND \\ WILLIAM J. FRY, M.D. \\ Section of General Surgery, Department of Surgery, University of \\ Michigan Medical Center, Ann Arbor, Michigan 48104
}

Submitted for publication December 1, 1975

Renovascular occlusive lesions account for the most common form of surgically correctable hypertension. Technical advances in vascular reconstructive surgery have increased the likelihood of renal parenchymal preservation and amelioration of hypertension in patients having renal artery stenoses. Proper selection of patients alleged to have renovascular hypertension for operative treatment is the most important determinant of a successful result.

Measurements of systemic and renal vein renin activity have been used to define the functional significance of suspected renal artery occlusive lesions. Ischemic kidney renin hypersecretion associated with contralateral renin suppression has recently evolved as a potentially reliable index of surgical curability [24]. The present investigation was undertaken to determine the discriminatory value of two expressions of excessive plasma renin activity. The reliability of systemic:renin indices (RSRI) and renal vein renin ratios (RVRR) in predicting responses to operation for remedial renovascular hypertension was assessed.

\section{MATERIALS AND METHODS}

Eighty-five patients with renovascular hypertension, in whom renin activity was

\footnotetext{
'Supported by the University of Michigan Peripheral Vascular Research Fund. Presented at the Ninth Annual Meeting of the Association for Academic Surgery, Minneapolis, Minnesota, November 13, 1975.

${ }^{2}$ Correspondence to Dr. Stanley, University Hospital, 1405 East Ann Street, Ann Arbor, Mich. 48104.
}

measured and results of surgical therapy established, provided the basis for study. Arterial fibrodysplasia affected renal arteries of 68 patients. The 61 females and 7 males with this disease ranged in age from 5 to 58 $\mathrm{yr}$ (mean $34.5 \pm 12.1)^{3}$. The 17 remaining patients, 14 males and 3 females, exhibited arteriosclerotic renal artery stenoses. They ranged in age from 37 to $64 \mathrm{yr}$ (mean $50.0 \pm$ 8.6). Without respect to existence of hemodynamically or functionally important lesions, bilateral renal artery disease was evident in 51 cases. Marked blood pressure elevation, averaging $206 \pm 32 / 120 \pm 16 \mathrm{~mm}$ $\mathrm{Hg}$, was noted in these patients upon first recognition of hypertension. Rigorous medical therapy failed to establish normal diastolic pressures prior to operative intervention in any patient.

Primary surgical procedures in the present experience included 83 renal artery reconstructions and two nephrectomies (including one partial nephrectomy for an arteriovenous malformation). Clinical responses following operation were carefully defined and adhered to during the study. Patients were classified cured when blood pressures were $150 / 90 \mathrm{~mm} \mathrm{Hg}$ or less for a minimum of 6 months, during which time no antihypertensive medications were administered. Patients were considered improved if normotensive with drug therapy or when diastolic pressures were at least $15 \%$ lower than preoperative levels, but less than 110

${ }^{3}$ All variances reported as \pm 1 SD. 
$\mathrm{mm} \mathrm{Hg}$. Failures included instances where diastolic pressures greater than $90 \mathrm{~mm} \mathrm{Hg}$ represented less that $15 \%$ decrease from preoperative levels, as well as those cases in whom diastolic pressures were greater than $110 \mathrm{~mm} \mathrm{Hg}$. Four failures who might otherwise have been included in this study were eliminated, as failure appeared to be the consequence of operative errors unassociated with patient selection. Applying these criteria to the present series, 51 patients were classified cured and 34 improved.

Radioimmunoassay or bioassay of renin activity from systemic arterial or peripheral venous samples, as well as from renal vein samples provided a means of evaluating renin secretion. Accuracy has not been significantly altered during conversion from pressor bioassays (angiotensin II), utilized early in this experience, to the present use of radioimmunoassay (angiotensin I) techniques. Stimulation of the renin-angiotensin system at the time of study was usually accomplished by restricting sodium intake and administering natriuretic drugs for $48-72 \mathrm{hr}$ prior to sampling. Most patients were studied after they had been in the upright position for approximately $2 \mathrm{hr}$. Variations in patient preparation did exist among the presently reported patients, although specific renin activities could not be correlated with specific inconsistencies. Renal vein renin ratios ( $R$ VRR), represented by ischemic kidney renin activity/contralateral kidney renin activity, were calculated in all 85 patients. Renal:systemic renin indices (RSRI), defined as (individual renal renin activity - systemic renin activity)/systemic renin activity, were determined in 33 patients. Differences in RVRR and RSRI among cured and improved patients were compared utilizing Student's $t$ test and $\chi^{2}$ statistical analyses.

\section{RESULTS}

Characterization of clinical groupings (cured-improved). The mean age of cured patients $(35.9 \pm 13.6 \mathrm{yr})$ was remarkably similar to that of patients classified improved (39.6 $\pm 11.7 \mathrm{yr})$. Hypertension among cured patients (mean 205 $\pm 35 / 118 \pm 11 \mathrm{~mm} \mathrm{Hg}$ ) was not statistically different from that affecting improved patients $(209 \pm 28 / 128 \pm$ $16 \mathrm{~mm} \mathrm{Hg}$ ). Unilateral renal artery disease, detected arteriographically, affected 28 of 51 cured patients, but only 6 of 34 improved patients. This difference proved statistically significant $(P<0.01)$. Presence of this contralateral disease, however, was rarely believed contributory to the hypertensive state.

Renal vein renin ratios (Fig. 1). The mean RVRR among cured patients was $2.08 \perp$ 0.71 . This was statistically indistinguishable from the mean RVRR of $2.01 \pm 1.41$ in the improved group. The RSRI was available in two of six cured patients exhibiting RVRR less than 1.4. In each case, the RSRI would have supported a prediction of curability. Five of eight improved patients having renin ratios less than 1.4 had known RSRI, and in each instance the limitation of surgical therapy was confirmed by the indices.

RVRR associated with unilateral disease $(2.10 \pm 0.94)$ did not vary significantly from that occurring with bilateral lesions $(2.02 \pm$

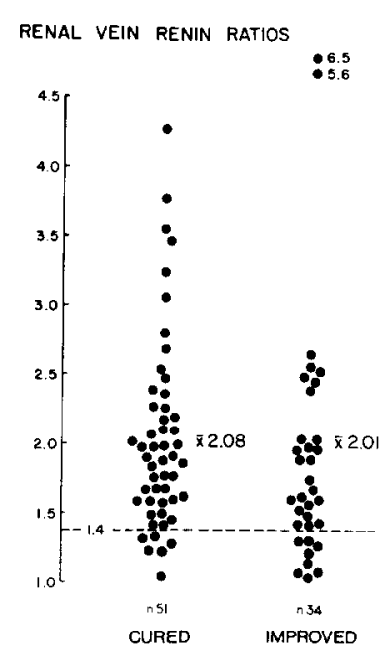

FIG. 1. Differential renal renin activity, comparing ischemic kidney to contralateral kidney. Statistical differences were not demonstrated between mean renal vein renin ratios among cured and improved cases $(2.08$ and 2.01 , respectively). 
0.87). Another observation deserving comment concerns the lack of statistically significant differences of RVRR in cases exhibiting collateral vessels circumventing stenotic lesions compared to those not demonstrating collaterals. The mean RVRR in the presence of collaterals was $1.88 \pm 0.62$, compared to $2.22 \pm 1.33$ in the absence of collateral vessels. The proportion of cured:improved patients with RVRR being less than $1.4(6: 8)$ 1.4-1.9 (21:13) 2.0-2.9 (18:11) and greater than $3.0(6.2)$ did not reveal better results in patients having greater RVRR.

Renal:systemic indices (Fig. 2). Ischemic kidney RSRI was decidedly greater than that of the contralateral kidney RSRI in both cured and improved categories. Differences among cured cases, comparing ischemic RSRI $(1.03 \pm 0.51)$ to the contralateral RSRI $(0.09 \pm 0.13)$ proved highly significant $(P<0.001)$. Similarly, differences in improved patients between ischemic RSRI $(1.18 \pm 1.07)$ and a contralateral RSRI $(0.49$ $\pm 0.62)$ were significant $(P<0.05)$. Dissimilarities could not be documented statistically between the ischemic kidney RSRI of the cured (1.03) and improved (1.18) groups. The mean contralateral RSRI in these two clinical categories $(0.09$ and 0.49 , respectively) proved to be significantly different $(P<0.01)$. Suppressed contralateral renin secretion in cured patients served to differentiate them from improved patients.

None of the three improved patients exhibiting marked contralateral renin suppression demonstrated ischemic kidney renin hypersecretion. In two of these cases, a RVRR greater than 1.4 implicated the presence of functionally important renal ischemia. Similarly, both cured patients in this experience failing to exhibit renin hypersecretion had an abnormal RVRR. Among all patients of the series in whom RSRI were available, RVRR varied among cured (1.97 $\pm 0.55)$ and improved $(1.51 \pm 0.36)$ groups. These differences proved statistically significant $(P<0.05)$. Unilateral versus bilateral disease did not appear to influence RSRI.

\section{DISCUSSION}

Numerous criteria have been utilized to improve the preciseness with which renovascular hypertension is recognized. The diagnosis is established only when salutory

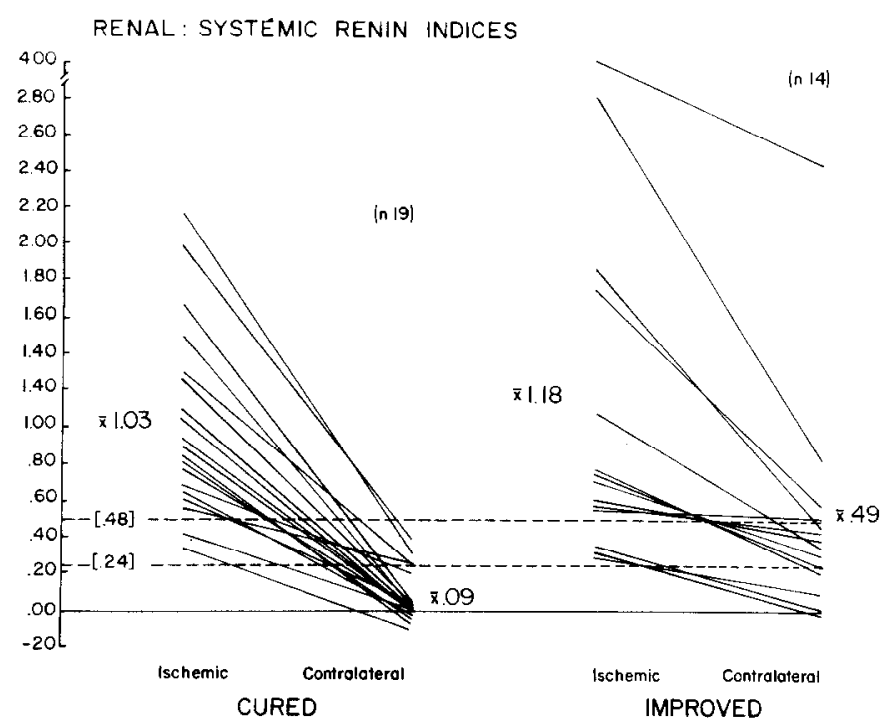

FIG. 2. Individual renal renin activity compared to systemic renin activity. Mean ischemic kidney hypersecretion in cured versus improved groups ( 1.03 and 1.18, respectively) did not prove to be statistically different. Evidence of contralateral suppression of renin activity was obvious in the cured $(0.09)$ group and nonexistent in the improved (0.49) group. Differences in the degree of suppression in these instances proved significant $(P<0.01)$. 
effects on blood pressure follow technically successful operations. Preoperative determinations of peripheral and renal blood pressor activity may be the most reliable means of identifying patients who will benefit from surgical therapy. Renin, the rate-limiting factor in angiotensin generation, is unequivocally involved in pathogenic mechanisms of clinical renovascular hypertension. Abnormal volume-sodium regulation may cause blood pressure elevations in differing forms of onekidney hypertension, but the vasoconstrictive renin-angiotensin system seems most important in initiating and maintaining twokidney hypertension associated with unilateral renal ischemia $[4,13]$. Although peripheral renin activity may appear normal, elevations of renal vein renin activity reflect increased renin secretion, not just altered concentrations secondary to decreased renal blood flow [27].

Variations in technique of specimen sampling, differences in assay methodology, and divergent interpretations of data have resulted in a number of misconceptions concerning renin and renovascular hypertension. The influence of patient preparation, including changes in posture $[5,16]$, sodium depletion $[17,23]$, and acute reductions in blood pressure [12], cannot be ignored in evaluating the existing literature. The source of blood for systemic plasma renin activity assays (peripheral arterial versus infrarenal inferior vena cava or peripheral venous) is not an important variable [20]. Absolute renin activity in peripheral $[2,5,8,9,22]$ or renal blood $[1$, $7,10]$ from patients having documented renovascular hypertension has not proven as reliable a prognostic indicator as previously believed. Correlation of peripheral renin activity with urinary excretion of sodium under controlled conditions has afforded a remarkably reliable means of documenting hyperreninemia associated with surgically correctable renovascular hypertension [13, 24]. Increased renin activity in the suprarenal inferior vena cava [14] is more likely to be elevated than peripheral activity but has not received favor as a diagnostic test.
Inability to suppress plasma renin activity has been suggested in assessing the significance of increased renin activity [25]. Comparisons of renal renin activity from both kidneys $[1-3,6-10,15-18,21-23,26,27]$ and comparisons of peripheral to renal renin activity $[3,12,17,18,21,22,24,26]$ have proven most important in evaluation of hypertensive patients suspected of renovascular origin.

Differential renal vein renin activity. The assumption that an RVRR greater than 1.5 reflects abnormal renin secretion is somewhat arbitrary. In a well-conceived and well-executed study of essential hypertensive patients, Sealey and her colleagues reported theoretical variance of individual renal renin activity of 1.0 to 1.48 times that occurring peripherally [19]. Compounding this physiologic disparity with that attributed to assay techniques [20], an RVRR much in excess of 1.48 (i.e., greater than 1.89 ) is necessary to assure existence of abnormal renin secretion. Overlooking technical problems, RVRR have been reported greater than 1.5 in $13 \%$ and more than 1.63 in $5 \%$ of the nonrenal hypertensives [19].

Rigid diagnostic criteria using unusually high RVRR lessens false positives. This practice entails a correspondingly higher incidence of false negative results with exclusion of potentially curable patients. In the current study, where an RVRR greater than 1.4 was considered abnormal, 14 of the 85 patients benefiting from operative therapy had normal ratios. Excepting many inconsistencies associated with interpretation of RVRR, the differential secretion rate comparing individual kidneys continues to be an important diagnostic study. However, as a discriminatory tool, RVRR has no value in distinguishing cured from improved subgroups. Mean RVRR in these latter categories (2.08 and 2.01, respectively), although distinctly abnormal, were not statistically different.

It is appealing, but difficult, to correlate specific RVRR levels with the outcome of treatment [21]. $\chi^{2}$ analysis of results failed to 
document any association between benefits (improved versus cured subgroups) within cells defined by renin ratios: (a) less than 1.4 , (b) 1.4-1.9, (c) 2.0-2.9, or (d) greater than 3.0.

Mean RVRR of 2.10 associated with unilateral disease did not vary statistically from that of 2.02 occurring with bilateral renal artery disease. A unilateral or bilateral designation refers to arteriographically demonstrable disease. Very few bilateral lesions proved hemodynamically or functionally significant. An elevated (nonsuppressed) contralateral RSRI may prove such occult lesions to be more important than previously thought. RVRR may appear normal in these instances, but renal renin secretion might be abnormally high.

Evolution of collateral vessels circumventing stenoses has been suggested to cause a reversion of RVRR towards normal [6]. Although this observation has occurred in patients with unilateral lesions, it has not been noted with bilateral disease [21]. Statistically significant differences in RVRR associated with collaterals $(1.88 \pm 0.62)$ in comparison to those without demonstrable collaterals $(2.22 \pm 1.33)$ could not be verified in the present study. Inability to validate differences in these two groups probably resides in wide variation in RVRR associated with demonstrable collaterals. The hypothesis that renal vein renin ratios revert toward unity as collateral vessels develop is a logical consideration but requires further documentation to be accepted.

Relation of individual renal to systemic renin activity. Recognition that renin clearance is proportional to arterial renin levels in hypertensive patients without renovascular causes is important in understanding renin kinetics [19]. Beneficial responses to operation occur regularly when ischemic kidney renin hypersecretion is associated with contralateral suppression in patients with renovascular hypertension [24]. Although individual renal vein renin activity may vary considerably from peripheral renin activity, statistical analysis of individual renin:systemic activity reveals a constant 1.24 ratio [19]. The total renal:systemic ratio accordingly is 1.48 . This is true over a wide range of circulating renin levels although with very high levels an individual ratio of 1.34 was observed [19]. Similar findings have been reported by others $[12,26]$. The validity of ischemic renal renin hypersecretion (RSRI > 0.48) and contralateral renin suppression (RSRI $<0.24$ to 0.0 ) in discriminating between cured and improved responses to operation receives support from the present investigation. The mean RSRI of 1.03 in cured patients and 1.18 among improved patients both reflect hypersecretion. When contralateral suppression appears complete, diminished effective renal plasma flow to the ipsilateral kidney may be estimated $[13,19$, 24]. Differences in the mean contralateral RSRI in the cured group (RSRI $=0.09$ ) compared to that of the improved group $($ RSRI $=0.49)$ proved highly significant. Persistently elevated blood pressure in improved cases following removal or revascularization of ischemic kidneys is predictable when nonsuppressed secretion from the contralateral kidney exists. Limited response to operation in three patients exhibiting contralateral suppression in the improved group perhaps reflects normal renin activity from the ipsilateral, and allegedly, ischemic kidney.

Acute stimulation (sampling in the upright position, etc.) of the renin-angiotensin system, utilized in the past to unmask differential renal vein activity, alters the steady state claimed necessary to evaluate renal:systemic renin activity adequately [19, 24]. Simultaneous acquisition of blood specimens, as advocated by some [13], was not performed in the present experience. Sequential sampling from the more diseased kidney first, followed by contralateral kidney and peripheral (infrarenal inferior vena cava) sampling will lessen false positive results [24]. Despite inconsistencies in preparation and sampling methods in the present study, renal:systemic renin indices were surprisingly constant and reflected with a high 
degree of accuracy the potential for cure or improvement following operation. Variations of renin activity as determined by bioassay compared to that measured by radioimmunoassay techniques do exist; however, these differences are constant [20] and should not affect calculation of either RVRR or RSRI.

Most important of all findings in the present study was the fact that documentation of renin suppression (contralateral RSRI approaching zero) provided a reliable index of curability in patients with renovascular hypertension. Renin hypersecretion has been recognized as important in establishing the diagnosis of renovascular hypertension [3, 12, 18, 21, 24], but the significance of contralateral suppression has been overlooked in all but a few studies [17, $18,21,22,24,26]$. Correlation of peripheral plasma renin activity to urinary sodium holds diagnostic promise but necessitates careful balanced studies in patients whose antihypertensive medications have been withheld.

Renal vein renin ratios are nondiscriminatory in differentiating cured from improved patients among renovascular hypertensives benefiting from operative therapy. Renal: systemic renin indices documenting contributions from both kidneys to circulating renin levels afford a means of predicting the degree of benefit anticipated following operation. Data generated from the present investigation expands upon interactions of the renin-angiotensin system and lends support to evolving tenets concerning this vasoconstrictive form of surgically correctable hypertension.

\section{ACKNOWLEDGMENT}

The authors wish to acknowledge the contributions to this work by George W. Williams, Ph.D., Department of Biostatistics, University of Michigan School of Public Health.

\section{REFERENCES}

1. Amsterdam, E. A., Couch, N. P., Christlieb, A. R., Harrison, J. H., Crane, C., Doerzinsky, S. J., and Kickler, R. B. Renal vein renin activity in the prognosis of surgery for renovascular hypertension. Amer.J. Med. 47:860, 1969.
2. Bath, N. M., Gunnells, J. C., and Robinson, R. R. Plasma renin activity in renovascular hypertension. Amer.J. Med. 45:381, 1968.

3. Bourgoigne, J., Kurz, S., Catanzaro, F. J., Serirat, P., and Perry, H. M. Renal venous renin in hypertension. Amer. J. Med. 48:332, 1970.

4. Brunner, H. R., Kirshman, J. D., Sealey, J. E., and Laragh, J. H. Hypertension of renal origin: Evidence for two different mechanisms. Science 174:1344, 1971.

5. Cohen, E. L., Rovner, D. R., and Conn, J. W. Postural augmentation of plasma renin activity. Importance in diagnosis of renovascular hypertension. J. Amer. Med. Ass. 197:973, 1966.

6. Ernst, C. B., Bookstein, J. J., Montie, J., Baumgartel, E., Hoobler, S. W., and Fry, W. J. Renal vein renin ratios and collateral vessels in renovascular hypertension. Arch. Surg. 104:496, 1972.

7. Fitz, A. Renal venous renin determinations in the diagnosis of surgically correctable hypertension. Circulation 36:942, 1967.

8. Gunnells, J. C., McGuffin, W. L., Johnsrude, I., and Robinson, R. R. Peripheral and renal venous plasma renin activity in hypertension. Ann. Intern. Med. 71: $555,1969$.

9. Hussain, R. A., Gifford, R. W., Stewart, B. H., Meaney, T. F., McCormack, L. J., Vidt, D. G., and Humphrey, D. C. Differential renal venous renin activity in diagnosis of renovascular hypertension. Review of 29 cases. Amer. J. Cardiol. 32:707, 1973.

10. Judson, W. E. and Helmer, O. M. Diagnostic and prognostic values of renin activity in renal venous plasma in renovascular hypertension. Hypertension 13:79, 1965.

11. Juncos, L. I., Strong, C. G., and Hunt, J. C. Prediction of results of surgery for renal and renovascular hypertension. Arch. Intern. Med. 134:655, 1974.

12. Kaneko, Y., Ikeda, T., Takeda, T., and Ueda, H. Renin release during acute reduction of arterial pressure in normotensive subjects and patients with renovascular hypertension. J. Clin. Invest. 46:705, 1967.

13. Laragh, J. H., Sealey, J. E., Buhler, F. R., Vaughan, E. D., Brunner, H. R., Gavras, H., and Baer, L. The renin axis and vasoconstriction volume analysis for understanding and treating renovascular and renal hypertension. Amer. J. Med. 58:4, 1975.

14. Meyer, P., Ecoiffier, J., Alexandre, J. M., Devaux, C., Guize, L., Menard, J., Biron, P., and Milliez, P. Prognostic value of plasma renin activity in renovascular hypertension. Circulation 36:570, 1967.

15. Michelakis, A. M., Foster, J. H., Liddle, G. W., Rhamy, R. K., Kuchel, O., and Gordon, R. D. Measurement of renin in both renal veins. Its use in diagnosis of renovascular hypertension. Arch. Intern. Med. 120:444, 1967.

16. Michelakis, A. M., and Simmons, J. Effect of posture on renal vein renin activity in hypertension. Its implications in the management of patients with re- 
novascular hypertension. J. Amer. Med. Ass. 208:659, 1969.

17. Pawsey, C. G. K., Vandongen, R., and Gordon, $R$. D. Renal venous renin ratio in the diagnosis of renovascular hypertension: Measurement during active secretion of renin. Med.J. A ust. 58:121, 1971.

18. Schambelan, M., Glickman, M., Stockigt, J. R., and Biglieri, E. G. Selective renal-vein renin sampling in hypertensive patients with segmental renal lesions. N. Engl. J. Med. 290:1153, 1974.

19. Sealey, J. E., Buhler, F. R., Laragh, J. H., and Vaughan, E. D. The physiology of renin secretion in essential hypertension. Estimation of renin secretion rate and renal plasma flow from peripheral and renal vein renin levels. Amer. J. Med. 55:391, 1973.

20. Sealey, J. E., Gerten-Banes, J., and Laragh, J. H. The renin system: Variations in man measured by radioimmunoassay or bioassay. Kidney Int. 1:240, 1972.

21. Stanley, J. C., and Fry, W. J. Renovascular hypertension secondary to arterial fibrodysplasia in adults. Criteria for operation and results of surgical therapy. A rch. Surg. 110:922, 1975.
22. Stockigt, J. R., Collins, R. D., Noakes, C. A., Schambelan, M., ard Biglieri, E. G. Renal-vein renin in various forms of renal hypertension. Lancet 1:1194, 1972.

23. Strong, C. G., Hunt, J. C., Sheps, S. G., Tucker, R. M., and Bernatz, P. E. Renal venous renin activity. Enhancement of sensitivity of lateralization by sodium depletion. Amer. J. Cardiol. 27:602, 1971.

24. Vaughan, E. D., Buhler, F. R., Laragh, J. H., Sealey, J. E., Baer, L., and Bard, R. H. Renovascular hypertension: Renin measurements to indicate hypersecretion and contralateral suppression, estimate renal plasma flow, and score for surgical curability. Amer. J. Med. 55:402, 1973.

25. Warren, D. J., and Ferris, T. F. Renin secretion in renal hypertension. Lancet 1:159, 1970.

26. Winer, B. M., Lubbe, W. F., Simon, M., and Williams, J. A. Renin in the diagnosis of renovascular hypertension. Activity in renal and peripheral vein plasma. J. Amer. Med. Ass. 202:121, 1967.

27. Woods, J. W., and Michelakis, A. M. Renal vein renin in renovascular hypertension. Arch. Intern. Med. 122:392, 1968. 